\title{
Highly effective photon-to-cooling thermal device
}

\author{
Yanpei Tian' ${ }^{1}$, Lijuan Qian², Xiaojie Liv ${ }^{1}$, Alok Ghanekar ${ }^{3}$, Gang Xiao ${ }^{2}$ \& Yi Zheng ${ }^{1 *}$ \\ Photon-to-cooling phenomenon relies on the atmospheric transparency window to dissipate heat \\ from the earth into outer space, which is an energy-saving cooling technique. This work demonstrates \\ a highly effective aluminized Polymethylpentene (PMP) thin-film thermal structure. The emissivity of \\ aluminized PMP thin films matches well to the atmospheric transparency window so as to minimize \\ parasitic heat losses. This photon-to-cooling structure yields a temperature drop of $8.5 \mathrm{~K}$ in comparison \\ to the ambient temperature and a corresponding radiative cooling power of $193 \mathrm{~W} / \mathrm{m} 2$ during a one-day \\ cycle. The easy-to-manufacture feature of an aluminized PMP thin film makes it a practically scalable \\ radiative cooling method.
}

Cooling technologies are substantial for energy-saving buildings and thermal management applications. Current cooling approaches rely mostly on compressors and fluid circulation systems with complex mechanical apparatus and high energy consumption. Passive cooling systems such as photon-to-cooling technology provides an alternative approach as energy conserving devices owing to their capability to operate without external energy input $^{1-4}$. Radiative cooling depends on the high transparency of earth's atmosphere at mid-infrared wavelengths $(7.8 \mu \mathrm{m} \sim 13 \mu \mathrm{m})^{5,6}$ and it corresponds to the selective optical absorption of polymer films in this range, such as Polyvinyl chloride (PVC) $)^{7}$, Polyvinyl fluoride (PVF) ${ }^{8-12}$, and Polymethylpentene (PMP), also known as TPX ${ }^{13,14}$. Due to its low cost, high effectiveness and flexibility to industrial productions, polymer films are highly promising materials for photon-to-cooling applications. Though all the emission spectra of the abovementioned polymer films cover the atmospheric transparency window, PMP has a better performance due to its higher emittance near $8 \mu \mathrm{m}^{13}$. The optical properties of PMP from near-infrared to mid-infrared wavelengths have not been well reported in the literature. It is significant and necessary to investigate optical properties, such as complex refractive indices of PMP free-standing films for residential and commercial radiative cooling applications.

Various methods have been employed for investigating the refractive index of thin films, such as refractometry and ellipsometry ${ }^{15-17}$. Refractometry is an very accurate method to measure the complex refractive index of ultra-thin film samples with a thickness from a few angstroms to several micrometers ${ }^{18}$. However, it loses accuracy for optically thick samples when the phase shift is greater than several $2 \pi^{19}$. Furthermore, ellipsometry is typically conducted in the reflection setup by measuring the change in polarization as the incident light interacts with the materials of interest ${ }^{20}$, and it requires that thin films are deposited on a smooth highly reflective substrate. It is commonly known that this method is not suitable for thin films of tens or hundreds of micrometers that are commercially available as free-standing films. In this work, refitting the reflectance spectrum of thin films with various thicknesses according to Lorentz-Drude has been proven to be a reliable and accurate approach to estimate refractive indices of materials of interest $\mathrm{t}^{21-23}$.

PMP is a transparent thermoplastic polymer of 4-Methyl-1-pentene repeating units ${ }^{24}$. It has a smallest density of $0.83 \mathrm{~g} / \mathrm{cm}^{3}$ in all the existing polymers. The transmittance of PMP in the visible region is slightly lower than Poly(methyl methacrylate) (PMMA), which is often used as a lightweight alternative material to glass ${ }^{25}$. The high emissivity of PMP between $7 \mu \mathrm{m}$ and $13 \mu \mathrm{m}$ is attributed to its functional groups and their corresponding molecular vibrations under infrared light irradiation. Consequently, when PMP is aluminized (approximately $250 \mathrm{~nm}$ thin) on its backside, it displays an extremely high reflectance in both visible and near-infrared regions while it exhibits high emittance matching perfectly with the atmospheric transparency window ${ }^{13}$. These advantageous optical properties with its relatively high mechanical strength ${ }^{26}$ which ensures PMP standout to be applied for scalable photon-to-cooling applications ${ }^{14}$.

In this paper, free-standing PMP thin films of various thicknesses are manufactured using the hot-press technique. For the first time, the complex refractive indices of PMP are determined by refitting the transmittance spectra using the Lorentz-Drude model and reported in near-/mid-infrared regions ranging from $0.9 \mu \mathrm{m}$ to

\footnotetext{
${ }^{1}$ Department of Mechanical and Industrial Engineering, Northeastern University, Boston, MA, 02115, USA. ${ }^{2}$ Department of Physics, Brown University, Providence, RI, 02912, USA. ${ }^{3}$ Artech LLC, Morristown, NJ, 07960, USA. *email:y.zheng@northeastern.edu
} 


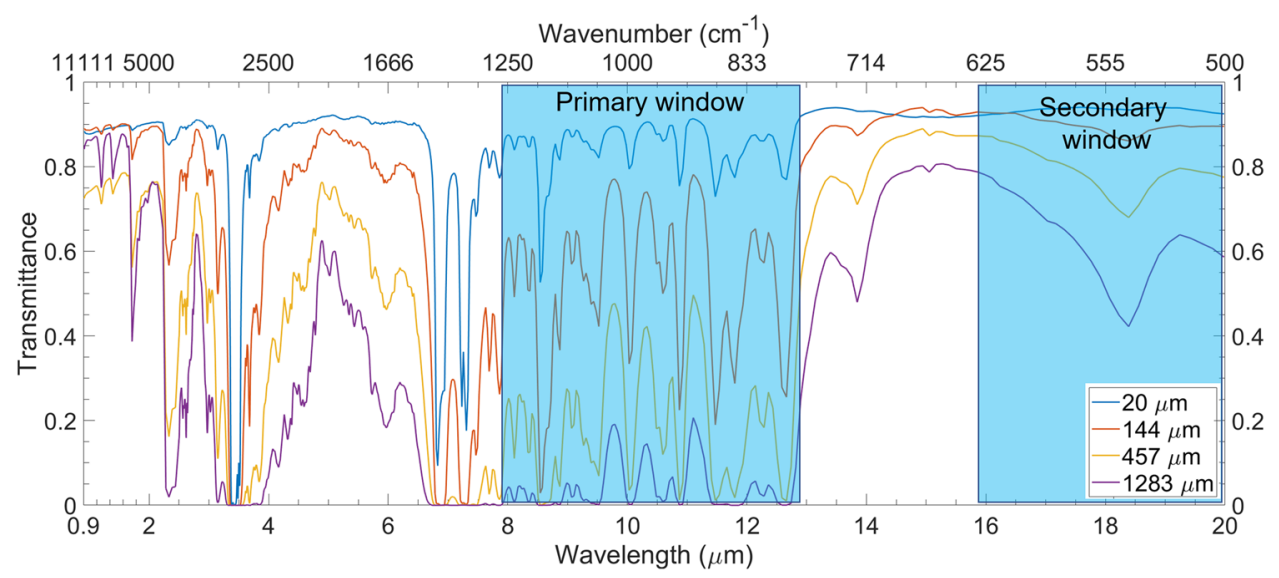

Figure 1. Transmittance measurement of PMP free-standing films with various thicknesses with two atmospheric transparency windows.

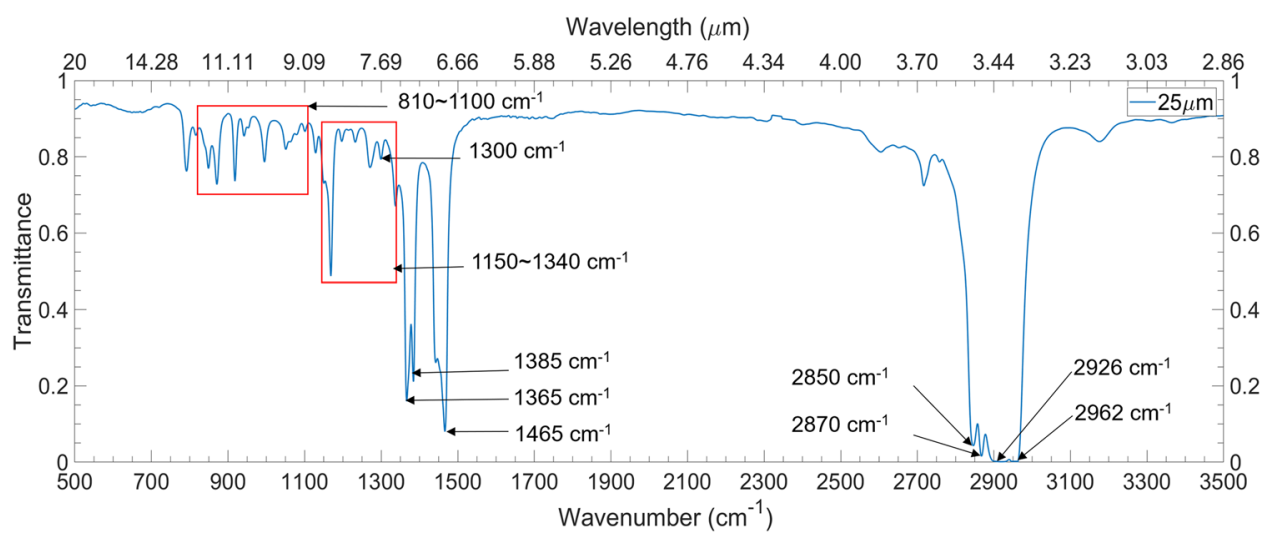

Figure 2. Transmittance measurement of a $20 \mu \mathrm{m}$ PMP free-standing film showing the various functional groups and their corresponding molecular vibration modes.

$20 \mu \mathrm{m}$. To validate the predicted refractive indices of PMP thin films, the transmittance and reflectance spectra are calculated using extracted refractive indices and compared with experimental data. The thermal performance of fabricated aluminized PMP radiative coolers is investigated for passive daytime cooling during a 24-hour cycle. A temperature drop of $8.5 \mathrm{~K}$ in comparison to ambient temperature and corresponding radiative cooling power of $193 \mathrm{~W} / \mathrm{m}^{2}$ during a one-day cycle are achieved to validate the high performance of photon-to-cooling thermal structures.

\section{Results}

Transmittance spectra analysis. Eight samples of PMP free-standing films with thicknesses of $20 \mu \mathrm{m}$, $48 \mu \mathrm{m}, 100 \mu \mathrm{m}, 144 \mu \mathrm{m}, 199 \mu \mathrm{m}, 386 \mu \mathrm{m}, 457 \mu \mathrm{m}$, and $1283 \mu \mathrm{m}$ are prepared by the hot-press method. Transmittance spectra of the $1283 \mu \mathrm{m}$ thick samples show a high absorptance, which match two atmospheric transparency windows (nearly unity absorptance in the primary atmospheric window from $8 \mu \mathrm{m}$ to $13 \mu \mathrm{m}$, and around half in the secondary atmospheric window from $16 \mu \mathrm{m}$ to $22 \mu \mathrm{m}$ ), as shown in Fig. 1. The primary window contributes $\sim 90 \%$ of the total radiative cooling power at near-ambient temperature due to much higher transmittance than the second atmospheric window 6 . The absorptance of the PMP films rise within these two atmospheric transmission windows with the increasing thickness of PMP films. FTIR spectroscopy is executed to identify different molecular structures and compositions in samples through the transmission spectra or attenuated total reflection (ATR) mode ${ }^{27,28}$. Both methods can display the distinct absorption bands, however, ATR is often used to measure surface properties relying on the total reflectance of evanescent waves and has a penetration depth of $1 \mu \mathrm{m}$ or $2 \mu \mathrm{m}$ depending on the ATR crystal material ${ }^{29}$.

It is obvious that the transmission spectrum analysis method is more suitable for much thicker PMP films. Distinct absorption peaks are clearly visible in PMP film transmittance spectra when the thickness is less than $50 \mu \mathrm{m}$ since the absorption peaks merge as shown in the transmission spectra of thicker PMP films, such as $457 \mu \mathrm{m}$ or $1283 \mu \mathrm{m}$ thick samples in Fig. 1 . While the thinner films show clearly distinct absorption peaks, thicker films display broadband absorptions, however, they cannot illustrate all the necessary functional groups or chemical bonds of PMP. It is reasonable that the transmittance spectrum of the $20 \mu \mathrm{m}$ film (Fig. 2) is selected to analyze 
the relationship between highly absorptance atmospheric windows and the chemical bonds of PMP. Evidently, there exist two different absorptions peaks: strong absorption peaks lie around $1400 \mathrm{~cm}^{-1}$ and $2900 \mathrm{~cm}^{-1}$, and weak absorption peaks lie in $810 \mathrm{~cm}^{-1} \sim 1100 \mathrm{~cm}^{-1}$ and $1150 \sim 1340 \mathrm{~cm}^{-1}$. The most notable absorption peaks around $2900 \mathrm{~cm}^{-1}$ are owing to the symmetric or asymmetric stretch vibrations of $\mathrm{CH}_{2}$ and $\mathrm{CH}_{3}$ : asymmetric stretch vibrations of $\mathrm{CH}_{2}$ and $\mathrm{CH}_{3}$ bonds at $2850 \mathrm{~cm}^{-1}(3.51 \mu \mathrm{m})$ and $2962 \mathrm{~cm}^{-1}(3.38 \mu \mathrm{m})$, respectively; symmetric stretch vibrations of $\mathrm{CH}_{2}$ and $\mathrm{CH}_{3}$ at $2926 \mathrm{~cm}^{-1}(3.42 \mu \mathrm{m})$ and $2870 \mathrm{~cm}^{-1}(3.48 \mu \mathrm{m})$, respectively. The strong absorption peak at $1465 \mathrm{~cm}^{-1}(6.83 \mu \mathrm{m})$ denotes the asymmetric scissoring bending vibrations of $\mathrm{CH}_{3}$ bond. Normally, the symmetric scissoring bending vibrations of $\mathrm{CH}_{3}$ bond are located at $1375 \pm 5 \mathrm{~cm}^{-1}(7.26 \mu \mathrm{m})^{30}$. However, when two or three $\mathrm{CH}_{3}$ groups are attached to the same carbon atom, the symmetrical scissoring bending vibration of $\mathrm{CH}_{3}$ will couple and cause the absorption band to split into two distinct peaks. For PMP, there are two $\mathrm{CH}_{3}$ groups that are connected to one carbon atom, so the symmetrical scissoring bending vibration of $\mathrm{CH}_{3}$ divides into two separated absorption peaks: $1365 \mathrm{~cm}^{-1}(7.32 \mu \mathrm{m})$ and $1385 \mathrm{~cm}^{-1}(7.22 \mu \mathrm{m})$. Since these absorption peaks resulting from strong bonds vibrations, the absorptance approaches to unity even though the thickness of PMP films is small. However, the weak absorption peaks are situated at $810 \mathrm{~cm}^{-1} \sim 1100 \mathrm{~cm}^{-1}$ $(9.09 \mu \mathrm{m} \sim 12.34 \mu \mathrm{m})$ and $1150 \mathrm{~cm}^{-1} \sim 1340 \mathrm{~cm}^{-1}(7.46 \mu \mathrm{m} \sim 8.69 \mu \mathrm{m})$. The corresponding absorption peaks increase with the thickness of PMP films. The $\mathrm{CH}_{3}$ wagging bending vibrations and $\mathrm{CH}_{2}$ out-of-plane wagging bending vibrations illustrate the weak absorptions peaks at $810 \mathrm{~cm}^{-1} \sim 1100 \mathrm{~cm}^{-1}$ and $1150 \mathrm{~cm}^{-1} \sim 1340 \mathrm{~cm}^{-1}$, respectively. These absorption peaks in the $20 \mu \mathrm{m}$ PMP film are attributed to surface phonon modes ${ }^{31}$ corre- $^{-}$ sponding to different vibration modes.

Refitting of complex refractive indices. Complex refractive indices of PMP are extracted by refitting measured transmittance spectra to calculated transmittance data. Six samples (thickness: $20 \mu \mathrm{m}, 48 \mu \mathrm{m}, 144 \mu \mathrm{m}$, $199 \mu \mathrm{m}, 386 \mu \mathrm{m}$, and $1283 \mu \mathrm{m}$ ) of PMP films are chosen to extract the complex refractive indices, which are used to calculate transmission spectra of other two samples of thickness $100 \mu \mathrm{m}$ and $457 \mu \mathrm{m}$ for the validation of the extracted refractive indices of PMP films.

Lorentz-Drude oscillator model for dielectric function of PMP is employed to calculate the refitted transmittance spectrum, which is given by ${ }^{32}$

$$
\varepsilon(\omega)=\varepsilon_{\infty}+\sum_{k=1}^{N} \frac{s_{k}}{1-\left(\frac{\omega}{\omega_{k}}\right)^{2}-j \Gamma_{k}\left(\frac{\omega}{\omega_{k}}\right)}
$$

Here, $s_{k}, \omega_{k}, \Gamma_{k}$ and $j$ are the strength, resonant frequency, damping factor of $k$ th Lorentz-Drude oscillator and the imaginary unit, respectively. $N$ such oscillators are assumed. $\varepsilon_{\infty}$ is the contribution from higher frequencies. The complex refractive indices $\sqrt{\varepsilon \mu}=n+j \kappa$, where $\varepsilon$ and $\mu$ are the permittivity and permeability, respectively. $n$ and $\kappa$ are the refractive index and extinction coefficient, respectively. Since PMP is a non-magnetic polymer, its permeability $\mu$ is 1 . The generalized transmissivity is given by

$$
\tau=\frac{1}{2}\left[\left|T^{T E}\right|^{2}+\left|T^{T M}\right|^{2}\right]
$$

where $T^{T E}$ and $T^{T M}$ are effective transmission coefficients at the given angle of incidence for transverse electric (TE) and transverse magnetic (TM) polarization, respectively. In this work, a thin film structure of air-PMP-air is considered for the normal transmission calculation and measurement. The generalized transmission coefficient for the interface between air and PMP can be expressed $\mathrm{as}^{33}$

$$
T^{(\beta)}=\frac{T_{1,2}^{(\beta)} e^{j k_{1 z} d}}{1-R_{1,2}^{(\beta)} \widetilde{R}_{2,3}^{(\beta)} e^{2 j k_{2 z} d}}
$$

where $T_{1,2}^{(\beta)}$ and $R_{1,2}{ }^{(\beta)}$ are the Fresnel transmission and reflection coefficient at the interface between layer 1 (air) and layer 2 (PMP film), respectively. $\widetilde{R}_{2,3}^{(\beta)}$ is the Fresnel reflection coefficient at the interface between layer 2 (PMP) and layer 3 (air). $\beta=s$ (or $p$ ) is for TE (or TM) polarization, $d$ is the thickness of PMP film. $k_{i z}=\sqrt{\varepsilon_{i}(\omega) \omega^{2} / c^{2}-k_{\rho}^{2}}$ is the normal component of the wave vector in air and PMP film, $\varepsilon_{i}(\omega)$ is the permittivity of air $\left(i=1, \varepsilon_{1}(\omega)=1\right)$ and PMP $(i=2)$ as a function of angular frequency $\omega, c$ is the speed of light in the vacuum and $k_{\rho}=\sin (\theta) \omega / c . \theta$ is the incident angle of beam to PMP films. For this case, the incident angle for transmission measurement is $0^{\circ}$.

Dielectric function of PMP can be extracted by tuning several oscillator parameters in order to match the measured transmittance spectra of these six samples. An optimization procedure is used to minimize the error between measured and refitted spectra. The error between these two spectra is given by

$$
\begin{aligned}
\delta= & \left.\sum_{i=1}^{M}\left[T_{m}-T_{r}\right]^{2}\right|_{20 \mu m}+\left.\sum_{i=1}^{M}\left[T_{m}-T_{r}\right]^{2}\right|_{48 \mu m}+\left.\sum_{i=1}^{M}\left[T_{m}-T_{r}\right]^{2}\right|_{144 \mu m} \\
& +\left.\sum_{i=1}^{M}\left[T_{m}-T_{r}\right]^{2}\right|_{199 \mu m}+\left.\sum_{i=1}^{M}\left[T_{m}-T_{r}\right]^{2}\right|_{386 \mu m}+\left.\sum_{i=1}^{M}\left[T_{m}-T_{r}\right]^{2}\right|_{1283 \mu m}
\end{aligned}
$$


A

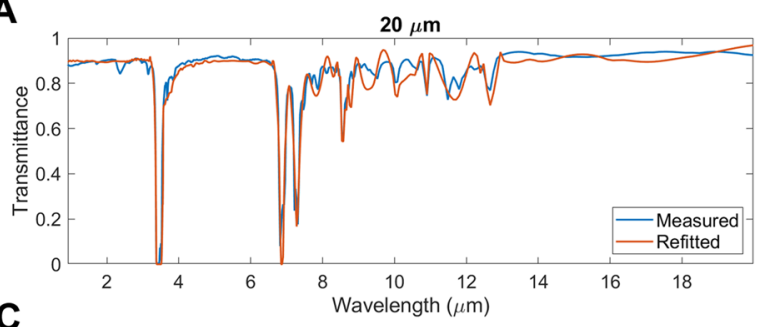

$144 \mu \mathrm{m}$

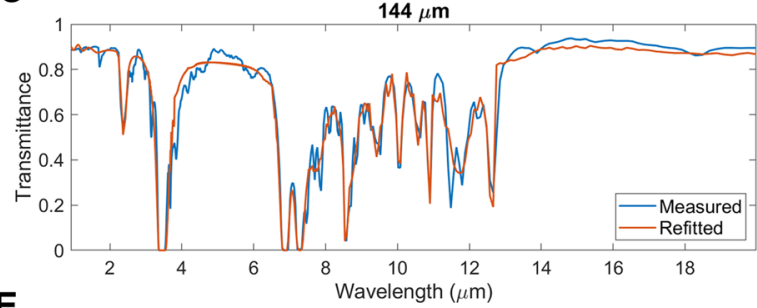

E

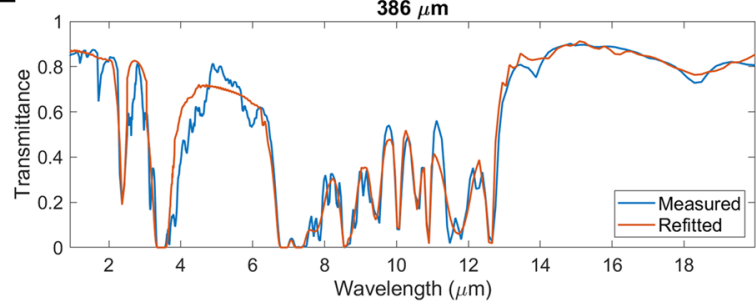

B

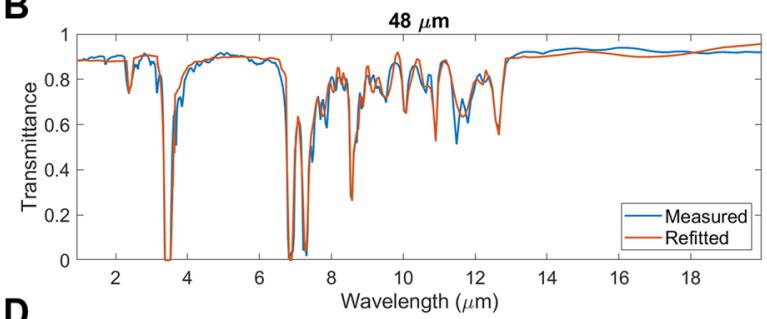

$199 \mu \mathrm{m}$

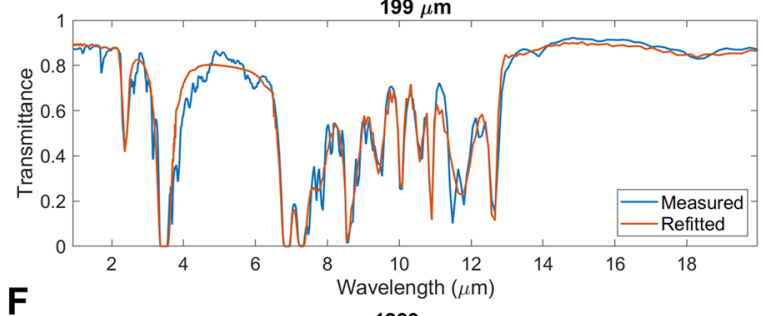

$1283 \mu \mathrm{m}$

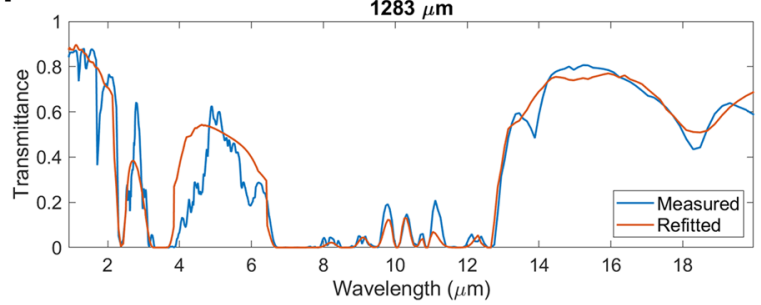

Figure 3. Transmittance measurement of PMP free-standing films of various thicknesses, (A) $20 \mu \mathrm{m},(\mathbf{B})$ $48 \mu \mathrm{m}$, (C) $144 \mu \mathrm{m}$, (D) $199 \mu \mathrm{m}$, (E) $386 \mu \mathrm{m}$, and (F) $1283 \mu \mathrm{m}$, in comparison with refitted spectra using the Lorentz-Drude oscillator model.

Here, $T_{m}$ and $T_{r}$ are measured and refitted values of transmittance, respectively. Index $i$ indicates different wavelengths over which the transmittance measurements are conducted. MATLAB based genetic algorithm is applied to obtain the final optimized resonant frequencies and achieve a good fit between measured and refitted spectra. It can be empirically found that 21 groups of oscillator parameters are suitable to approach the best fit. Comparisons of measured and refitted transmittance spectra are shown in Fig. 3A $(20 \mu \mathrm{m}), 3 \mathrm{~B}(48 \mu \mathrm{m}), 3 \mathrm{C}(144 \mu \mathrm{m}), 3 \mathrm{D}$ $(199 \mu \mathrm{m}), 3 \mathrm{E}(386 \mu \mathrm{m})$, and $3 \mathrm{~F}(1283 \mu \mathrm{m})$, which display good fits for all six PMP samples. Most of the absorption peaks for various PMP films are fitted using the oscillator parameters listed in Table 1 . The deviations between the measured and refitted spectrum increases as the thickness increases, which can be seen in Fig. $3 \mathrm{~F}$. This is because there are fewer absorption peaks in the sample of $1283 \mu \mathrm{m}$ compared with other samples, and it is hard to get a good fit using a set of same oscillator parameters. The refitting method optimizes these oscillator parameters which is corresponding to these absorption peaks to get a good fit between measured and refitted spectra. The real and imaginary parts of the complex refractive indices of PMP are shown in Fig. 4. The higher extinction coefficients $(\kappa)$ around $3.4 \mu \mathrm{m}\left(2900 \mathrm{~cm}^{-1}\right)$ and $6.8 \mu \mathrm{m}\left(1470 \mathrm{~cm}^{-1}\right)$ result from the strong stretch and bending vibrations of $\mathrm{CH}_{2}$ and $\mathrm{CH}_{3}$ bonds. The weak wagging bending vibrations of $\mathrm{CH}_{2}$ and $\mathrm{CH}_{3}$ bonds denote appearances of small peaks at extinction coefficients between $7.22 \mu \mathrm{m}$ to $12.34 \mu \mathrm{m}$, which corresponds to the primary atmospheric window.

Validation of extracted refractive indices. In order to validate the refitting procedure of PMP films, transmittance spectra are evaluated for $100 \mu \mathrm{m}$ and $457 \mu \mathrm{m}$ thick PMP samples using Eqs. (2) and (3) with the extracted complex refractive indices (Fig. 4). The measured and refitted transmittance spectra of PMP thin films with thicknesses of $100 \mu \mathrm{m}$ and $457 \mu \mathrm{m}$ show a well match as shown in Fig. 5A,B, respectively. These 12 main absorption peaks of these two curves occurs at the same wavelength. It confirms that the extracted refractive indices of PMP can be used to predict the optical properties of PMP related thermal photonic structures.

\section{Discussion}

PMP film based radiative cooler. Aluminized PMP films are easily manufactured structures serving as a radiative cooler. Magnetron sputtering technology is employed to deposit a $250 \mathrm{~nm}$ thin aluminum on the back of a $500 \mu \mathrm{m}$ PMP film, which is thick enough to block all the lights from visible to mid-infrared range ${ }^{34}$. The diffused FTIR reflectance measurements follow the procedures to be discussed in the method section. The emissivity of the radiative cooler of interest can be obtained from the reflectance measurement based on Kirchhoff's law by $\varepsilon=1-\tau-\rho$, here $\rho$ is the measured reflectance and transmittance $\tau$ is zero. As shown in Fig. 6 , the simulated emissivity curve of the sample matches well with the experimental result and it shows low emissivity in the solar radiation wavelength region and high emissivity in the two earth atmospheric transparency windows (highlighted regions in blue). Since the refitting method optimizes these oscillator parameters which correspond to 


\begin{tabular}{|c|c|c|c|c|}
\hline $\mathbf{k}$ & $\omega_{k}$ & $\lambda_{k}$ & $s_{k}$ & $\Gamma_{k}$ \\
\hline \multirow[t]{2}{*}{-} & $\left(\mathrm{cm}^{-1}\right)$ & $(\mu \mathrm{m})$ & - & - \\
\hline & & \multicolumn{2}{|c|}{$\varepsilon_{\infty}=1.4$} & \\
\hline 1 & 5415 & 1.84 & 9.907E-08 & \begin{tabular}{|l|}
$0.999 \mathrm{E}-00$ \\
\end{tabular} \\
\hline 2 & 4197 & 2.38 & $6.149 \mathrm{E}-05$ & $2.721 \mathrm{E}-02$ \\
\hline 3 & 3968 & 2.52 & $2.153 \mathrm{E}-07$ & 0.999E-00 \\
\hline 4 & 4050 & 2.47 & $2.655 \mathrm{E}-04$ & \begin{tabular}{|l|}
$2.935 \mathrm{E}-06$ \\
\end{tabular} \\
\hline 5 & 2863 & 3.49 & $7.570 \mathrm{E}-02$ & $1.822 \mathrm{E}-04$ \\
\hline 6 & 1818 & 5.50 & $1.756 \mathrm{E}-04$ & 3.010E-01 \\
\hline 7 & 1456 & 6.87 & $4.936 \mathrm{E}-03$ & \begin{tabular}{|l|}
$1.082 \mathrm{E}-03$ \\
\end{tabular} \\
\hline 8 & 1372 & 7.28 & $8.621 \mathrm{E}-04$ & 7.413E-03 \\
\hline 9 & 1290 & 7.75 & 3.161E-04 & \begin{tabular}{|l|}
$3.394 \mathrm{E}-02$ \\
\end{tabular} \\
\hline 10 & 1166 & 8.58 & 7.217E-03 & 3.727E-07 \\
\hline 11 & 1163 & 8.60 & $3.532 \mathrm{E}-03$ & 3.734E-05 \\
\hline 12 & 1153 & 8.67 & $3.227 \mathrm{E}-04$ & \begin{tabular}{|l|}
$1.968 \mathrm{E}-02$ \\
\end{tabular} \\
\hline 13 & 1061 & 9.42 & $1.638 \mathrm{E}-04$ & $1.635 \mathrm{E}-02$ \\
\hline 14 & 995 & 10.05 & $8.696 \mathrm{E}-05$ & \begin{tabular}{|l|}
$5.278 \mathrm{E}-03$ \\
\end{tabular} \\
\hline 15 & 946 & 10.57 & $9.078 \mathrm{E}-05$ & $1.026 \mathrm{E}-02$ \\
\hline 16 & 919 & 10.88 & $1.880 \mathrm{E}-04$ & $8.700 \mathrm{E}-04$ \\
\hline 17 & 853 & 11.72 & $5.537 \mathrm{E}-04$ & 2.874E-02 \\
\hline 18 & 804 & 12.44 & 7.769E-04 & 6.204E-05 \\
\hline 19 & 792 & 12.62 & $4.886 \mathrm{E}-03$ & 6.805E-05 \\
\hline 20 & 670 & 14.91 & $2.036 \mathrm{E}-03$ & $2.385 \mathrm{E}-07$ \\
\hline 21 & 542 & 18.45 & $1.105 \mathrm{E}-04$ & $4.915 \mathrm{E}-02$ \\
\hline
\end{tabular}

Table 1. Oscillator parameters of PMP used to refit the transmittance spectra.

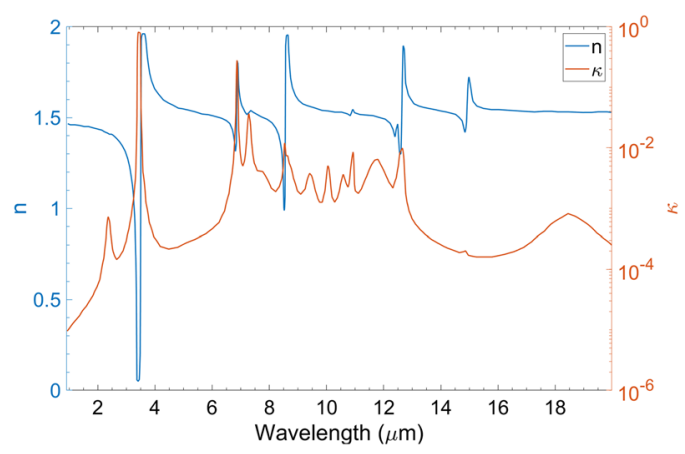

Figure 4. Real $(n)$ and imaginary $(\kappa)$ parts of the complex refractive indices of PMP extracted by refitting the transmittance spectra.

these absorption peaks to get a good fit between measured and refitted spectra, the discrepancy arises outside the primary atmospheric window, where most of these absorption peaks locates.

Thermal performance investigation. In order to demonstrate the photon-to-cooling capability of the radiative cooler under direct solar irradiation, its temperature variations are simulated by solving the thermal balance equation as expressed by ${ }^{35,36}$

$$
Q_{\text {total }}=Q_{\text {cooler }}\left(T_{\text {cooler }}\right)-Q_{\text {conv }}\left(T_{\text {amb }}, T_{\text {cooler }}\right)-Q_{\text {amb }}\left(T_{\text {amb }}\right)-Q_{\text {sun }}\left(T_{\text {cooler }}\right)
$$

It is supposed that the backside of the PMP cooler is insulated, we just consider the heat transfer between PMP cooler, air and outer space. Here, $Q_{\text {cooler }}$ is the net cooling power of the radiative cooler, $Q_{\text {conv }}$ is due to the natural air convective heat transfer, $Q_{a m b}$ is the incident thermal radiation from ambient, and $Q_{\text {sun }}$ stands for the incident solar irradiation absorbed by the radiative cooler. $T_{\text {cooler }}$ and $T_{a m b}$ are the temperatures of radiative cooler and ambient air, respectively. $Q_{\text {cooler }}$ can be determined as follows:

$$
Q_{\text {cooler }}\left(T_{\text {cooler }}\right)=A \int_{0}^{\infty} d \lambda I_{B B}\left(T_{\text {cooler }}, \lambda\right) \varepsilon\left(\lambda, \theta, \phi, T_{\text {cooler }}\right)
$$



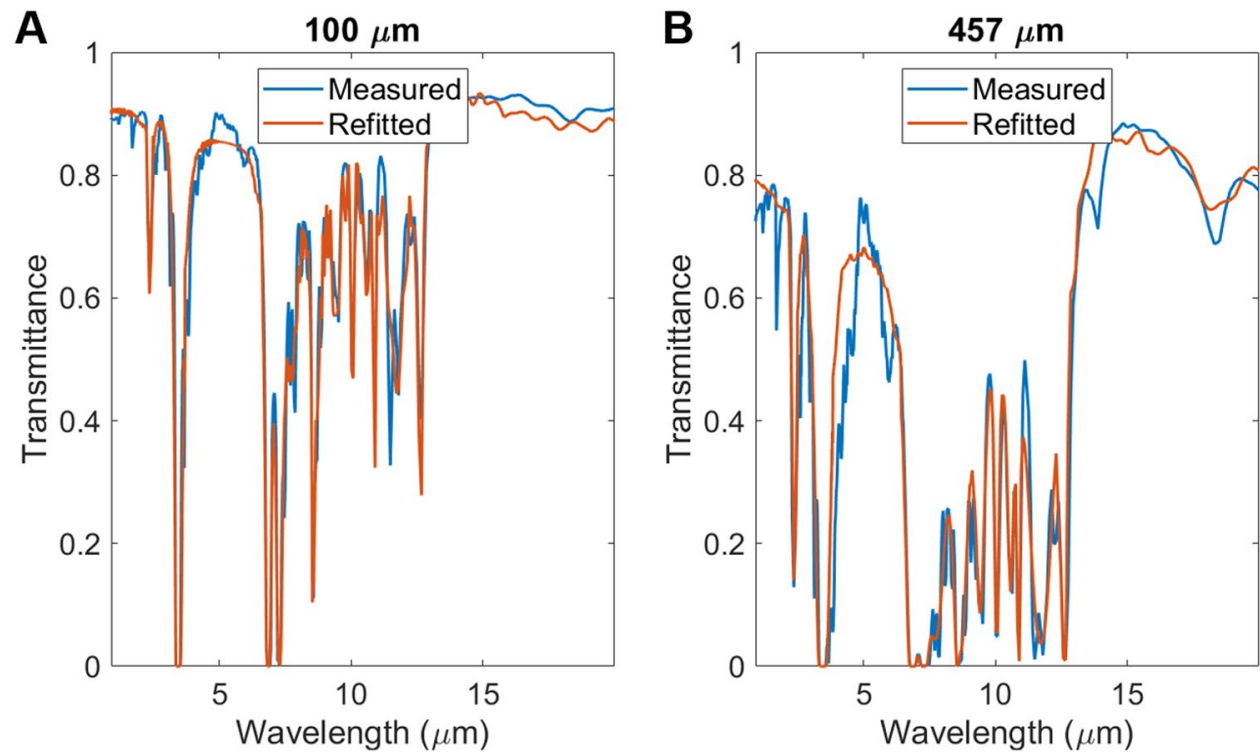

Figure 5. Validation of extracted complex refractive indices through comparisons between measured transmittance and refitted spectra for (A) $100 \mu \mathrm{m}$ and (B) $457 \mu \mathrm{m}$ thick PMP samples.

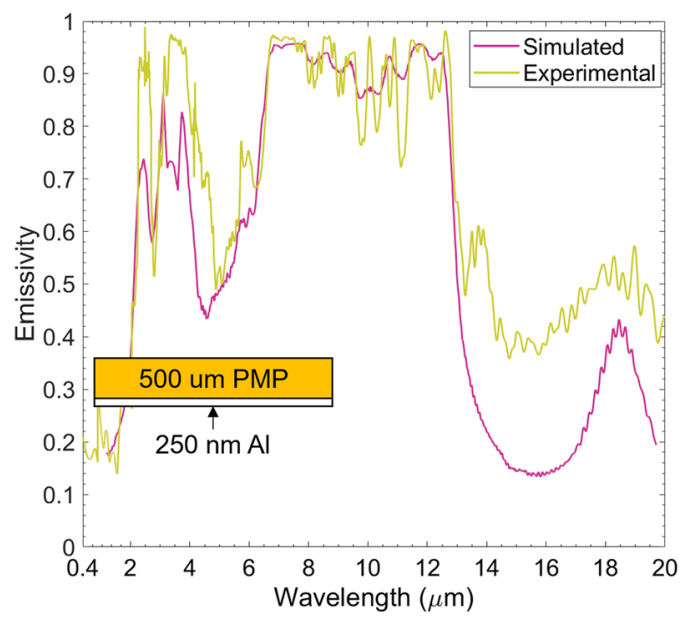

Figure 6. Measured and simulated emittance spectra of a $500 \mu \mathrm{m}$ PMP free standing film sputtered $250 \mathrm{~nm}$ thin aluminium on its backside.

where, $A$ is the area of radiative cooler, $I_{B B}\left(T_{\text {cooler }}, \lambda\right)=2 h c^{2} \lambda^{-5} \exp \left(h c / \lambda k_{B} T-1\right)^{-1}$ defines the spectral radiance of blackbody at a certain temperature $T$, where $h$ is the Planck's constant, $k_{B}$ is the Boltzmann constant, and $\lambda$ is the wavelength. $\varepsilon\left(\lambda, \theta, \phi, T_{\text {cooler }}\right)=\frac{1}{\pi} \int_{0}^{2 \pi} \mathrm{d} \varphi \int_{0}^{\pi / 2} \varepsilon_{\lambda} \cos \theta \sin \theta \mathrm{d} \theta$ is the temperature-dependent emissivity of radiative cooler $^{37}$. Here, the emissivity measured at room temperature $(298 \mathrm{~K})$ is taken into simulation, since it is assumed that the temperature variations of PMP and aluminum affect little on emissivity measurement. $\theta$ and $\phi$ are the azimuthal and latitudinal angles, respectively.

The parasitic heat transfer between the radiative cooler and ambient air is given by

$$
Q_{\text {conv }}\left(T_{a m b}, T_{\text {cooler }}\right)=A h_{a}\left(T_{a m b}-T_{\text {cooler }}\right)
$$

$h_{a}$ is the nonradiative heat transfer coefficient ranging from 2 to $8 \mathrm{Wm}^{-2} \mathrm{~K}^{-18,35,38}$. Here $h_{a}=8 \mathrm{Wm}^{-2} \mathrm{~K}^{-1}$ is set as natural air conduction and convection heat transfer to the radiative cooler. The absorbed power from incident thermal radiation from atmosphere $Q_{a m b}\left(T_{a m b}\right)$ is given by

$$
Q_{a m b}\left(T_{a m b}\right)=A \int_{0}^{\infty} \mathrm{d} \lambda I_{B B}\left(T_{a m b}, \lambda\right) \varepsilon\left(\lambda, \theta, \phi, T_{\text {cooler }}\right) \varepsilon(\lambda, \theta, \phi)
$$



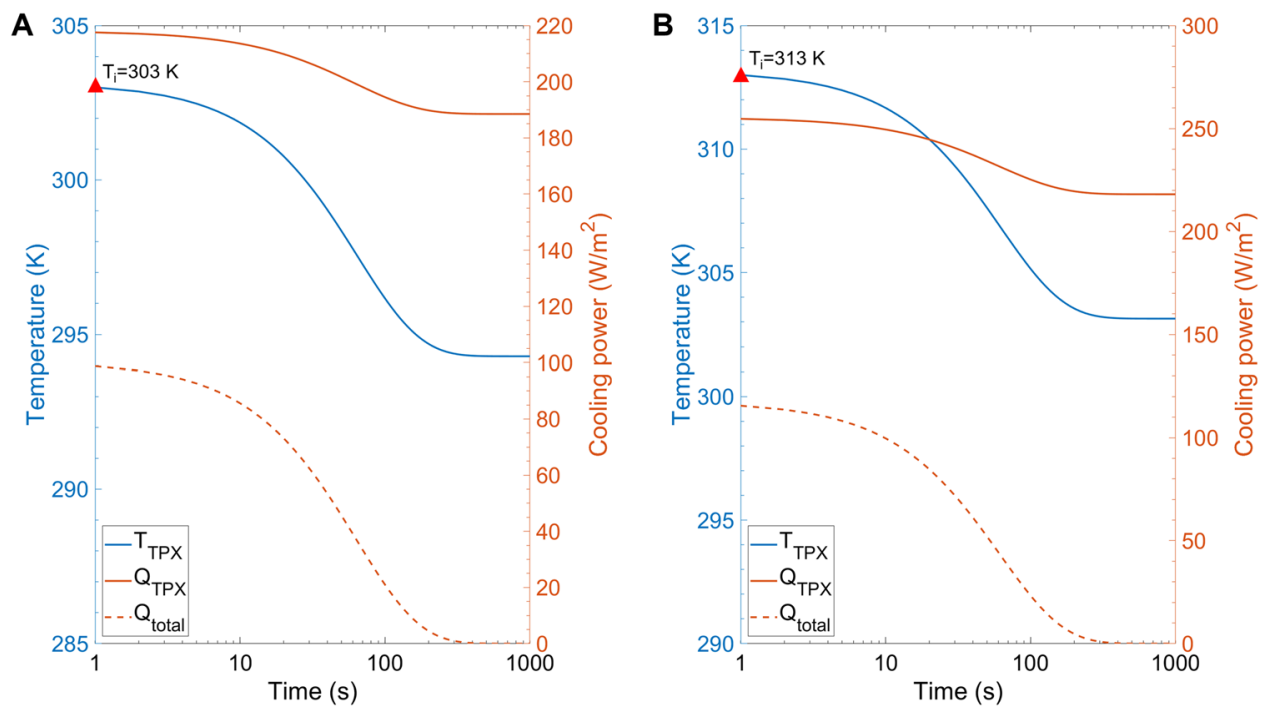

Figure 7. Transient thermal performance of the radiative cooler at different initial temperatures assumed to be the same as ambient temperature. Time-dependent evolution of $T_{P M P}, Q_{P M P}$ and $Q_{\text {total }}$ at (A) $303 \mathrm{~K}$ and (B) $313 \mathrm{~K}$ ambient temperature.

The absorptivity of the atmosphere, $\varepsilon(\lambda, \theta, \phi)$, is given by $1-\tau(\lambda, \theta, \phi)$. Here $\tau(\lambda, \theta, \phi)$ is the transmittance value of atmosphere obtained from MODTRAN $4^{39}$. Solar irradiation absorbed by the radiative cooler $Q_{\text {sun }}\left(T_{\text {cooler }}\right)$ is given by

$$
Q_{\text {sun }}\left(T_{\text {cooler }}\right)=A \int_{0}^{\infty} \mathrm{d} \lambda I_{A M 1.5}(\lambda) \varepsilon\left(\lambda, \theta_{\text {sun }}, T_{\text {cooler }}\right)
$$

Here, $I_{\mathrm{AM} 1.5}(\lambda)$ is the spectral irradiance intensity of solar irradiation at AM 1.5. $\varepsilon\left(\lambda, \theta_{\text {sun }}, T_{\text {cooler }}\right)$ is the temperature-dependent emissivity of radiative cooler, we take the measured data of the radiative cooler at room temperature into consideration.

The time-dependent temperature variations of the radiative cooler can be obtained by solving the following equation

$$
C_{\text {cooler }} \frac{d T}{d t}=Q_{\text {total }}\left(T_{\text {cooler }}, T_{\text {amb }}\right)
$$

Since the aluminum is sputtered on the PMP film, thermal contact resistance is negligible. The heat capacitance of the radiative cooler, $C_{\text {cooler }}$, consists of PMP $(500 \mu \mathrm{m})$ and aluminum $(250 \mathrm{~nm})$.

The transient temperature fluctuations of the radiative cooling system under two different ambient temperature conditions are simulated by solving Eq. (10), which is integrated to obtain the temperature evolution of radiative cooler as a function of time, as shown in Fig. 7. For each simulation, the initial temperature of the radiative cooler $\left(T_{i}\right)$ is assumed to be the same as the ambient temperature.

When the ambient temperature is $303 \mathrm{~K}$, the temperature of the radiative cooler, $T_{P M P}$, reduces and eventually reaches a thermal equilibrium temperature that is about $9 \mathrm{~K}$ below the ambient temperature because of the cooling effects of radiative cooler (blue solid curve in Fig. 7A). The photon-to-cooling performance of the radiative cooler $Q_{P M P}$ remains over $180 \mathrm{~W} / \mathrm{m}^{2}$ and reduces slightly with the evolution of time (orange solid curve in Fig. 7A). The net output of the radiative cooler $Q_{\text {total }}$ reduces and approaches zero when the cooler is at thermal equilibrium condition (orange dashed curve in Fig. 7A). Similarly, when the ambient temperature is $313 \mathrm{~K}$, as shown in Fig. 7B, the temperature of the radiative cooler eventually drops around $10 \mathrm{~K}$ below the ambient temperature. The cooling power $Q_{P M P}$ and net output $Q_{\text {total }}$ of the cooler follow the same behaviors as described in the previous case of the ambient temperature of $303 \mathrm{~K}$.

In the end, the radiative cooling temperature response is simulated under a real weather condition on July 20 , 2018, in Santa Clare, California ${ }^{40}$, which has a typical summer climate. Using the ambient temperature and solar illumination data of July 20,2018 ${ }^{41}$, as inputs of Eq. (5), the net radiative cooling power of radiative cooler and temperatures are simulated over a 24-hour period, as shown in Fig. 8. The ideal radiative cooler is set to be with unity emissivity in the atmospheric transparency window while it has zero emissivity at other wavelengths, and it yields a maximum temperature drop $\Delta \mathrm{T}$ of $10.6 \mathrm{~K}$. The inset of Fig. 8 displays the temperature difference between the radiative cooler and the ambient occurs and reaches thermal equilibrium in about $300 \mathrm{~s}$, then it fluctuates with the variations of ambient temperature. The temperature drops $\Delta \mathrm{T}$ at sunrise, noon, and sunset remain over $7 \mathrm{~K}$. It promises sufficient cooling effects. The maximum temperature difference of about $8.5 \mathrm{~K}$ occurs at around 3:30 PM when both the net radiative cooling power and the ambient temperature reach to the maximum ${ }^{42,43}$. 


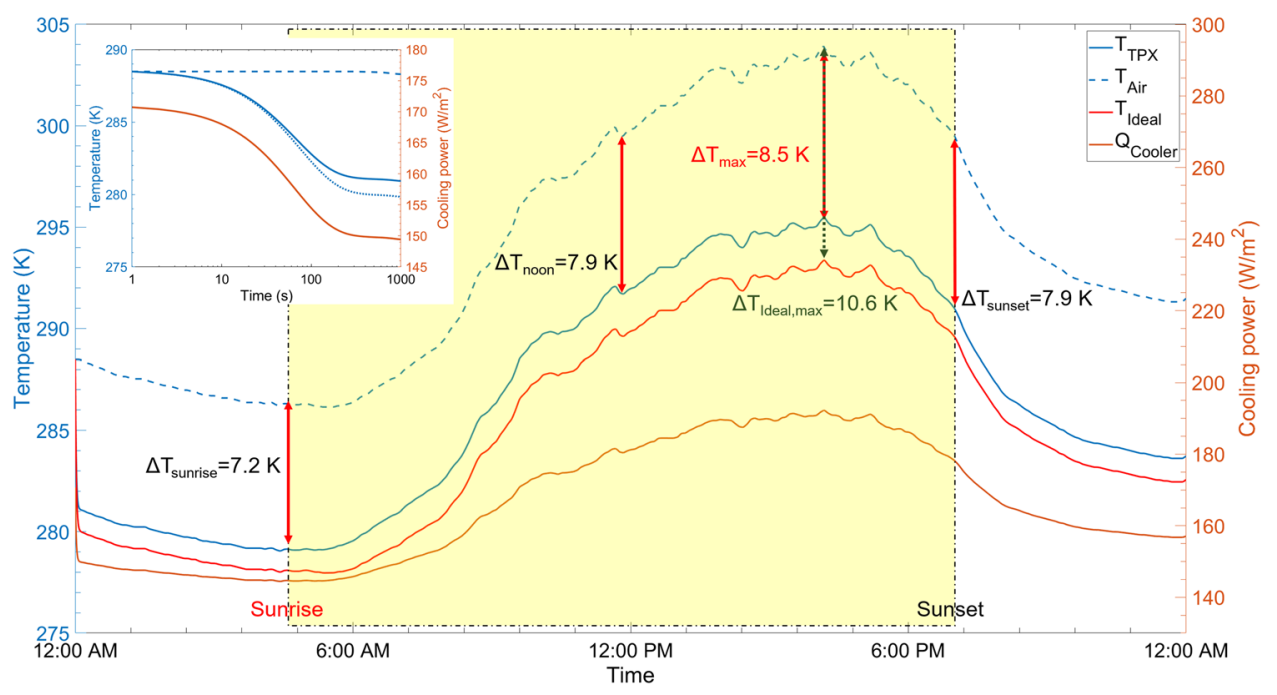

Figure 8. Left $y$-axis: thermal performance of the actual radiative cooler (blue solid curve) and the ideal one (blue dotted curve) over a 24-hour cycle at varying ambient temperatures (blue dashed curve). Right $y$-axis: net radiative cooling power (orange solid curve). Inset: Time-dependent evolution of thermal performance of a radiative cooler for the very beginning 1000 seconds.

A

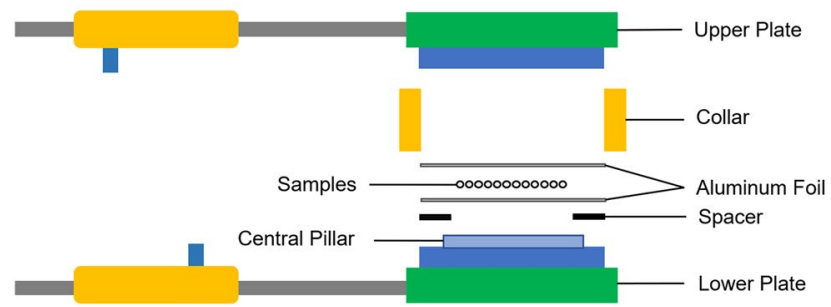

B

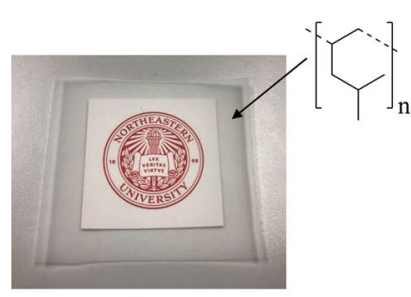

Figure 9. (A) Schematic of hot-press mold for thin film preparation. (B) The Northeastern University logo covered by a $76 \mu \mathrm{m}$ thick PMP film and the wireframe of PMP.

\section{Methods}

Sample preparation and instruments. PMP granules with a nominal size of $3 \mathrm{~mm}$ are purchased from GoodFellow, Inc. (USA) and sodium hydroxide $(\mathrm{NaOH})$ with a purity of $98 \%$ is supplied by Sigma-Aldrich Co. Inc. (USA). The electric auto hydraulic duel rosin press machine with PID temperature control of $1{ }^{\circ} \mathrm{C}$ accuracy is purchased from TechTongDa, Inc. (USA). The hot-press mold (Fig. 9A) is home built, and the spacer has an inner diameter of 1 inch and an outer diameter of 1.5 inches with various thicknesses. The aluminum target of $99.999 \%$ purity is purchased from Angstrom Sciences. The PMP sheet with a thickness of $500 \mu \mathrm{m}$ is purchased from CS Hyde Company (USA). The thickness of PMP thin films is measured by iGaging digital electronic micrometer with an accuracy of $\pm 4 \mu \mathrm{m}$.

PMP films preparations for transmittance measurement. First, turn on the hydraulic press machine and preheat the pressing plate above the melting temperature of PMP $\left(250^{\circ} \mathrm{C}\right)$, considering the heat resistance between different parts of the hot-press mold. All the parts of the hot-press mold are cleaned using successive washes of acetone and deionized water and dried with dry nitrogen gas, then put the lower platen, collar, the bottom aluminum foil, and spacer successively. A certain amount of PMP granules are put on the bottom aluminum foil at the center of the spacer according to the desired thickness and density of PMP films. After PMP granules melt, carefully put the top aluminum foil and the upper plate, then start to press with a pressure of 70 psi. 1 minute later, take out the hot-press mold and cool down to the room temperature. For the sake of obtaining intact films, put the PMP thin film with aluminum foils into $3 \mathrm{~mol} / \mathrm{L} \mathrm{NaOH}$ solution until the aluminum foils dissolve. Finally, take the PMP thin film out from $\mathrm{NaOH}$ solution and wash with deionized water, then put PMP thin film into a preheated $60^{\circ} \mathrm{C}$ convection oven for 20 minutes to release adsorbed water before transmittance spectra measurement. Through changing the amount of PMP granules and the thickness of spacers, PMP thin films of various thicknesses can be obtained, a PMP thin film with a thickness of $76 \mu \mathrm{m}$ is shown in Fig. 9B.

Aluminized PMP radiative cooler preparations. A $250 \mathrm{~nm}$ thin aluminum film is deposited on one side of the PMP sheet via a home-built high vacuum magnetron sputtering machine. The base pressure (vacuum 
A

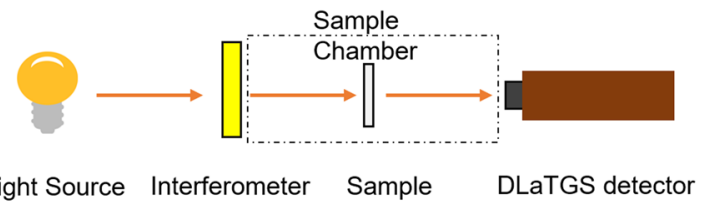

B
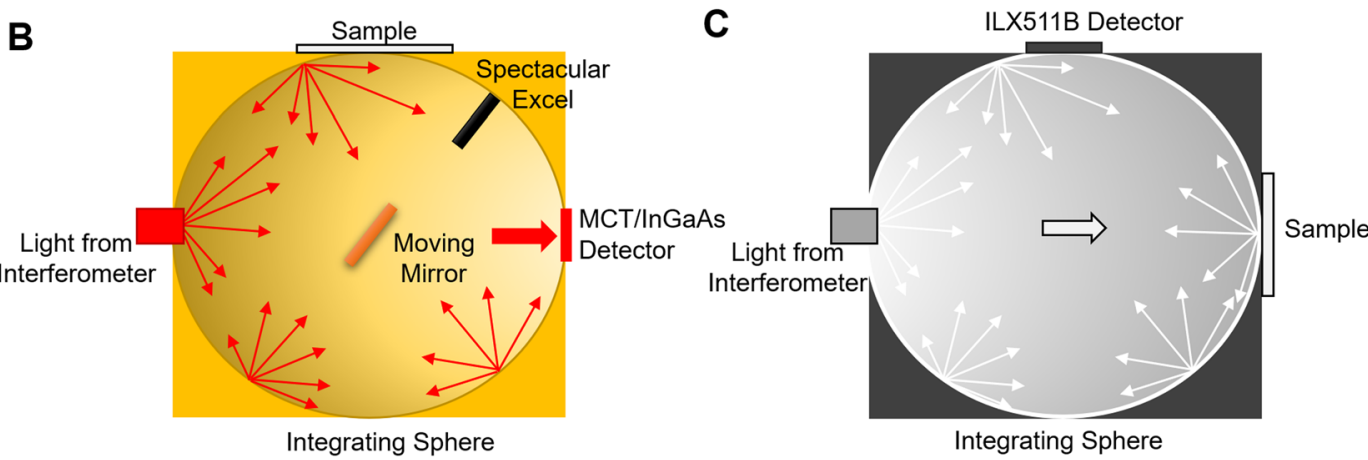

Figure 10. (A) Schematic of an experimental setup of the transmittance measurement. (B) Schematic of diffuse reflectance measurement accessory with an integrating sphere for near-infrared $(0.9 \mu \mathrm{m} \sim 2.5 \mu \mathrm{m})$ and midinfrared $(2.5 \mu \mathrm{m} \sim 20 \mu \mathrm{m})$ reflectance measurement. (C) Schematic of diffuse reflectance measurement accessory with an integrating sphere for Visible $(0.38 \mu \mathrm{m} \sim 0.74 \mu \mathrm{m})$ and near-infrared $(0.74 \mu \mathrm{m} \sim 0.85 \mu \mathrm{m})$ region measurements.

before sputtering starts) and Argon pressure during sputtering are $4.2 \times 10^{-4}$ mTorr and 0.87 mTorr, respectively. The aluminum thin film is deposited on the PMP sheet with a sputtering rate of $0.0838 \mathrm{~nm} / \mathrm{s}$ at $15 \mathrm{~W}$ DC power supply.

\section{Transmittance and Reflectance Measurement}

Transmittance measurement. The transmittance spectra of PMP thin films are measured with the experimental setup shown in Fig. 10A. The light source, interferometer, and Deuterated Lanthanum $\alpha$ Alanine doped TriGlycine Sulphate (DLaTGS) detector are located inside the Jasco 6600 Fourier Transform Infrared Spectroscopy (FTIR) spectrometer chamber. Jasco 6600 are equipped with halogen light source (from $0.9 \mu \mathrm{m}$ to $2.5 \mu \mathrm{m}$ ) and ceramic light source (from $2.5 \mu \mathrm{m}$ to $25 \mu \mathrm{m}$ ). The DLaTGS detector has a high accuracy from $0.9 \mu \mathrm{m}$ to $20 \mu \mathrm{m}$. The samples of PMP thin films are sandwiched between infrared sample cards with an aperture of $17 \mathrm{~mm}$. These samples are placed vertically to the optical path inside the sample chamber with a Jasco infrared cards holder. Each transmittance measurement includes a background measurement and a sample measurement. Scan rate is set to 32 with a resolution of $4 \mathrm{~cm}^{-1}$. Three measurements are taken for each sample and the average value of three measurements is taken as the final result for further analysis.

Diffuse reflectance measurement. Diffuse reflectance measurements of near-infrared and mid-infrared are characterized using FTIR spectrometer equipped with PIKE's diffuse reflectance accessory equipped with an integrating sphere, as shown in Fig. 10B. The accessory has a spherical shell with an inner surface coated with a diffuse reflective layer of gold and two infrared light detectors - indium gallium arsenide (InGaAs) (from $0.9 \mu \mathrm{m}$ to $2.5 \mu \mathrm{m}$ ) and mercury cadmium telluride (MCT) detector (from $2.5 \mu \mathrm{m}$ to $20 \mu \mathrm{m}$ and cooled by liquid nitrogen). The integrating sphere has three ports: one is for light from the interferometer and the other two are for the sample and MCT/InGaAs detector, respectively. The infrared light from the interferometer falls on the moving mirror. When the moving mirror is turned to face to diffuse gold, the spectrometer takes the reference measurement (background reflectance is assumed to be $100 \%$ ). The moving mirror can be switched to point at the sample with an incident angle fixed at $12^{\circ}$. The light reflected from the sample scatters at the inner surface of the integrating sphere and is captured by MCT/InGaAs detector. The scan rate is set to 64 with a resolution of $4 \mathrm{~cm}^{-1}$.

Diffused reflectance measurements of visible lights $(0.38 \mu \mathrm{m} \sim 0.74 \mu \mathrm{m})$ and near-infrared lights $(0.74 \mu \mathrm{m} \sim 0.85 \mu \mathrm{m})$ are characterized with Ocean Optics USB20000+ VIS-NIR-ES spectrometer equipped with a THORLABS 2-inch integrating sphere, as shown in Fig. 10C. This integrating sphere is inner-coated with a $1 \mathrm{~mm}$ thick PTFE based composite that has nearly unity reflectively within $0.25 \mu \mathrm{m} \sim 2.5 \mu \mathrm{m}$ wavelength range. The integrating sphere has three ports: one is for light from the Ocean Optics halogen light source HL-2000 and the other two are for the sample/reflectance reference and Sony ILX511B detector that covers the wavelength from $0.35 \mu \mathrm{m} \sim 1.0 \mu \mathrm{m}$, respectively. First, take the background spectrum using the end-cap with a coating of $1 \mathrm{~mm}$ thick reflective material on the port, then place samples at the same port for measuring the reflectance spectrum. For these diffuse reflectance measurement discussed above, three measurements are taken from three different locations on each sample, and the averaged data is considered as the final results for further analysis.

Received: 24 July 2019; Accepted: 11 November 2019;

Published online: 17 December 2019 


\section{References}

1. Florides, G. A., Tassou, S. A., Kalogirou, S. A. \& Wrobel, L. Review of solar and low energy cooling technologies for buildings. Renew. Sustain. Energy Rev. 6, 557-572 (2002).

2. Kim, D. \& Ferreira, C. I. Solar refrigeration options-a state-of-the-art review. Int. J. refrigeration 31, 3-15 (2008).

3. Khan, J. \& Arsalan, M. H. Solar power technologies for sustainable electricity generation-a review. Renew. Sustain. Energy Rev. 55, 414-425 (2016)

4. Smith, G. \& Gentle, A. Radiative cooling: Energy savings from the sky. Nat. Energy 2, 17142 (2017).

5. Hossain, M. M. \& Gu, M. Radiative cooling: principles, progress, and potentials. Adv. Sci. 3, 1500360 (2016).

6. Sun, X., Sun, Y., Zhou, Z., Alam, M. A. \& Bermel, P. Radiative sky cooling: fundamental physics, materials, structures, and applications. Nanophotonics 6, 997-1015 (2017).

7. Trombe, F. Perspectives sur l'utilisation des rayonnements solaires et terrestres dans certaines régions du monde. Rev. Gen. Therm 6, 1285 (1967).

8. Bartoli, B. et al. Nocturnal and diurnal performances of selective radiators. Appl. Energy 3, 267-286 (1977).

9. Catalanotti, S. et al. The radiative cooling of selective surfaces. Sol. Energy 17, 83-89 (1975).

10. Berdahl, P., Martin, M. \& Sakkal, F. Thermal performance of radiative cooling panels. Int. J. Heat Mass Transf. 26, 871-880 (1983).

11. Granqvist, C.-G. Radiative heating and cooling with spectrally selective surfaces. Appl. optics 20, 2606-2615 (1981).

12. Addeo, A. et al. Light selective structures for large scale natural air conditioning. Sol. Energy 24, 93-98 (1980).

13. Grenier, P. Réfrigération radiative. effet de serre inverse. Revue de Physique Appliquee 14, 87-90 (1979).

14. Zhai, Y. et al. Scalable-manufactured randomized glass-polymer hybrid metamaterial for daytime radiative cooling. Science $\mathbf{3 5 5}$, 1062-1066 (2017).

15. Xi, J.-Q. et al. Optical thin-film materials with low refractive index for broadband elimination of fresnel reflection. Nat. photonics 1, 176 (2007).

16. McCrackin, F. L., Passaglia, E., Stromberg, R. R. \& Steinberg, H. L. Measurement of the thickness and refractive index of very thin films and the optical properties of surfaces by ellipsometry. J. Res. Nat. Bur. Sec. A 67 (1963).

17. Baklanov, M., Mogilnikov, K., Polovinkin, V. \& Dultsev, F. Determination of pore size distribution in thin films by ellipsometric porosimetry. J. Vac. Sci. \& Technol. B: Microelectron. Nanometer Struct. Process. Meas. Phenom. 18, 1385-1391 (2000).

18. Tompkins, H. G. \& McGahan, W. A. Spectroscopic ellipsometry and reflectometry: a user's guide (Wiley, 1999).

19. Warenghem, M. et al. Combined ellipsometry and refractometry technique for characterisation of liquid crystal based nanocomposites. Rev. Sci. Instruments 83, 035103 (2012).

20. Tompkins, H. \& Irene, E. A. Handbook of ellipsometry (William Andrew, 2005).

21. Ghanekar, A., Ricci, M., Tian, Y., Gregory, O. \& Zheng, Y. Dynamic optical response of su-8 upon uv treatment. Opt. Mater. Express 8, 2017-2025 (2018).

22. Srinivasan, A., Czapla, B., Mayo, J. \& Narayanaswamy, A. Infrared dielectric function of polydimethylsiloxane and selective emission behavior. Appl. Phys. Lett. 109, 061905 (2016).

23. Tian, Y. et al. Near-infrared optics of nanoparticles embedded silica thin films. Opt. Express 27, A148-A157 (2019).

24. Lopez, L. C., Wilkes, G. L., Stricklen, P. M. \& White, S. A. Synthesis, structure, and properties of poly (4-methyl-1-pentene). J. Macromol. Sci. Part C: Polym. Rev. 32, 301-406 (1992).

25. Cariou, J.-M., Dugas, J., Martin, L. \& Michel, P. Refractive-index variations with temperature of pmma and polycarbonate. Appl. optics 25, 334-336 (1986).

26. Shin, S.-C. \& Yoon, M.-K. Application of tpx polymer membranes for the controlled release of triprolidine. Int. journal pharmaceutics 232, 131-137 (2002).

27. Smith, B. C. Fundamentals of Fourier transform infrared spectroscopy (CRC press, 2011).

28. Wang, X.-B. et al. Thermal uv treatment on su-8 polymer for integrated optics. Opt. Mater. Express 4, 509-517 (2014).

29. Wu, Y. et al. An ftir-atr investigation of in vivo poly (ether urethane) degradation. J. applied polymer science 46, 201-211 (1992).

30. Larkin, P. Infrared and Raman spectroscopy: principles and spectral interpretation (Elsevier, 2017).

31. Narayanaswamy, A., Mayo, J. \& Canetta, C. Infrared selective emitters with thin films of polar materials. Appl. Phys. Lett. 104, 183107 (2014).

32. Verleur, H. W. Determination of optical constants from reflectance or transmittance measurements on bulk crystals or thin films. JOSA 58, 1356-1364 (1968).

33. Chew, W. C. \& Chew, W. C. Waves and fields in inhomogeneous media, vol. 522 (IEEE press New York, 1995).

34. Tian, Y., Ghanekar, A., Liu, X., Sheng, J. \& Zheng, Y. Tunable wavelength selectivity of photonic metamaterials-based thermal devices. J. Photonics for Energy 9,032708 (2018).

35. Ono, M., Chen, K., Li, W. \& Fan, S. Self-adaptive radiative cooling based on phase change materials. Opt. express 26, A777-A787 (2018).

36. Yang, P., Chen, C. \& Zhang, Z. M. A dual-layer structure with record-high solar reflectance for daytime radiative cooling. Sol. Energy 169, 316-324 (2018).

37. Zhang, Z. Nano/Microscale Heat Transfer (McGraw-Hill, New York, 2007).

38. Raman, A. P., Anoma, M. A., Zhu, L., Rephaeli, E. \& Fan, S. Passive radiative cooling below ambient air temperature under direct sunlight. Nature 515, 540 (2014).

39. Berk, A. et al. Modtran4 radiative transfer modeling for atmospheric correction. In Optical spectroscopic techniques and instrumentation for atmospheric and space research III, vol. 3756, 348-354 (International Society for Optics and Photonics, 1999).

40. Weatherground. Weather conditions. https://www.wunderground.com/personal-weather-station/ dashboard?ID=KCASTANF2\#history/s20180720/e20180720/mdaily [Online; accessed 20-July-2018] (2018).

41. PVEDUCATION.ORG. Calculation of Solar Insolation. https://www.pveducation.org/pvcdrom/properties-of-sunlight/calculationof-solar-insolationy (2018).

42. Nilsson, T. M. \& Niklasson, G. A. Radiative cooling during the day: simulations and experiments on pigmented polyethylene cover foils. Sol. energy materials solar cells 37, 93-118 (1995).

43. Nilsson, T. M., Niklasson, G. A. \& Granqvist, C.-G. Solar-reflecting material for radiative cooling applications: Zns pigmented polyethylene. In Optical Materials Technology for Energy Efficiency and Solar Energy Conversion XI: Selective Materials, Concentrators and Reflectors, Transparent Insulation and Superwindows, vol. 1727, 249-262 (International Society for Optics and Photonics, 1992).

\section{Acknowledgements}

This project was supported in part by the National Science Foundation through grant numbers CBET-1836967, and National Aeronautics and Space Administration through grant number NNX15AK52A.

\section{Author contributions}

Y.T. prepared the PMP samples and wrote the manuscript with assistance from all the contributing authors. L.Q. performed the sputtering process for these samples. X.L. executed the MATLAB refitting process. A.G. assisted the PMP samples preparation and FTIR measurement. G.X. and Y.Z. supervised this project. 


\section{Competing interests}

The authors declare no competing interests.

\section{Additional information}

Correspondence and requests for materials should be addressed to Y.Z.

Reprints and permissions information is available at www.nature.com/reprints.

Publisher's note Springer Nature remains neutral with regard to jurisdictional claims in published maps and institutional affiliations.

(c) (i) Open Access This article is licensed under a Creative Commons Attribution 4.0 International License, which permits use, sharing, adaptation, distribution and reproduction in any medium or format, as long as you give appropriate credit to the original author(s) and the source, provide a link to the Creative Commons license, and indicate if changes were made. The images or other third party material in this article are included in the article's Creative Commons license, unless indicated otherwise in a credit line to the material. If material is not included in the article's Creative Commons license and your intended use is not permitted by statutory regulation or exceeds the permitted use, you will need to obtain permission directly from the copyright holder. To view a copy of this license, visit http://creativecommons.org/licenses/by/4.0/.

(c) The Author(s) 2019 